Notre Dame Journal of Formal Logic

Volume 40, Number 2, Spring 1999

\title{
Proposition and Tense
}

\author{
JAMES CARGILE
}

\begin{abstract}
McTaggart assumed (1) that propositions cannot change in truth value and (2) if (a) there is real change, then (b) events must acquire the absolute property of being present and then lose this property. He held that $\{1,2 b\}$ is an inconsistent set and thus inferred $\sim 2 \mathrm{a}$-that there is no real change. The $B$ theory rejects 2 and the $A$ theory rejects 1 . I accept 1, 2, 2a, and consequently, $2 \mathrm{~b}$, and argue that this is consistent. There is an absolute property of presentness, but we can never predicate this property without producing an assertion in which that property is "chronologized." If at $t$, we attribute presentness, we thereby attribute presentness-at- $t$. We can grasp the existence of an absolute property which we are unable to attribute absolutely.
\end{abstract}

Kant discusses a formulation of what he calls "the principle of contradiction": "It is impossible that anything should be and at the same time not be" 11. He objects that the term "impossible" is redundant, and more importantly, that the principle "as a purely logical principle, must not be limited in its application by time; and the beforementioned formula runs therefore counter to its very nature." Kant is not saying that the formula is not a necessary truth. Nor is he saying that there are no things such that they could at one time be and at another time not be, so that the formula for such things would require the temporal qualification he finds objectionable in a logical law. It is just that for such things as propositions, as intended in classical logic, we say simply that no such thing can be both true and not true, without needing any such qualification as "at the same time".

Temporally qualifying the principle of contradiction may suggest that it is about things that change truth value over time. Of course, the principle is about absolutely everything, including itself, and including sentences or "propositions" which change truth value over time. But it could be held that the principle does not require temporal qualification even for one who believes that propositions change truth value. It is a defensible view that "Nothing is both red and not red" is not refuted by the existence of a wall which is at one time red and at another, not. Similarly for all properties, whether or not they are "chronologized." Still, Kant's point has a stylistic basis.

Received September 29, 1998; revised March 1, 2000 
The formulation he criticizes suggests (even though it may not entail) the attitude that propositions, as properly intended in the classical rule that no proposition is both true and false, are of a nature such that we should add the qualifier "at the same time." We may consider whether this is true.

An example of something which might seem capable of being true at one time and false at another is:

A. The sundial is illuminated at present.

There may be a notion of "proposition" such that some such is associated with the sentence A in certain uses and, like the sentence, changes in truth value. But let us consider the proposition in the classical sense, as the entire content of what is said in a particular use of A.

Suppose someone uses A to make a statement at noon on Monday. At noon on Tuesday he asserts the sentence A again, in reference to the very same sundial, to the very same audience. We can keep the circumstances as similar as we like, except that there is a day separating the two assertions. The proposition conveyed by asserting the sentence A on Monday is MA, the one on Tuesday is TA. Question: Is MA = TA?

Someone who holds there is such a property as being present should feel some pressure to conclude that $\mathrm{MA}=\mathrm{TA}$. It would seem that both attribute the very same property, being illuminated at present, to the very same thing, the sundial. One could hold that in spite of this, they differ in content. But then it should be a problem to explain how they differ, for those who hold that there is a property of being present and thus a property of being both present and illuminated. Consider the following:

B. This case of the sundial's being illuminated is present.

Could this sentence B be used on Monday to make the same assertion as MA? What information would be conveyed by asserting one that would not be conveyed by asserting the other? One suggestion (due to an anonymous referee) is that use of "this case" signals that a direct reference by ostension is being made, and this is information not signaled by the use of A. On this account, the content of MA is conveyed by

MA*: The sundial's illumination has the property of presentness, whereas the content of MB is conveyed by

MB*: Whatever case of the sundial's illumination that is being demonstrated or ostended has the property of presentness.

The suggestion is that A could be used to refer to a case of a sundial being illuminated when the dial was not in the near vicinity and not being ostended, for example, if the speaker and audience were in the United States and the dial were in Egypt, while B could not properly be so used.

On the contrary, B could just as successfully be used to refer, from the vantage of the U.S., to a dial in Egypt. There would need to be some prior understanding that a sundial in Egypt is under discussion, perhaps concerned with various important consequences triggered by the exposure of that dial to light. Having described the results of a number of cases of illuminating the dial, perhaps by moving it outside or 
by artificially illuminating it, B could be used quite naturally and correctly to report that the illumination presently being discussed is in fact itself present.

A and B could indeed be used to say different things. But so can A itself or B itself on different occasions. We should make clear that we are concerned with one particular occasion on Monday and one particular occasion on Tuesday, with the same group of people gathered in the same way around the sundial. That the reference to the dial is "demonstrative" or "ostensive" could introduce the challenge of attempting a precise characterization of these notions. Whether such a project could be successful given the possible vagueness of those notions should not be an impediment to this present discussion of presentness. It would seem that the gathering we are trying to characterize would involve ostensive reference on both days as clearly as ostensive reference is ever achieved. The idea is then, that in just that context, A and B would be intersubstitutable. Someone could arrange that asserting B would signal something different from A. But following the standard meaning, A and B would convey the same assertion on Monday. That is, MA $=$ MB.

It seems clear that the assertion of B on Tuesday, $\mathrm{TB}$, would not convey the same information as MB. It would refer to a different case of the sundial's being illuminated. It is one thing for the sundial to be illuminated on Monday and another for it to be illuminated on Tuesday. It seems equally clear that TA $=\mathrm{TB}$. Assuming that $\mathrm{MA}=\mathrm{MB}$ and $\mathrm{TA}=\mathrm{TB}$ and that $\mathrm{MB}$ does not equal $\mathrm{TB}$ makes it inconsistent to hold that $\mathrm{MA}=\mathrm{TA}$. These plausible assumptions thus put pressure on one who holds there is a property of being present, since that makes it hard to deny that MA $=\mathrm{TA}$.

It might be objected at this point that what has been assumed to be clear is not so clear after all. For they are claims about the identity of propositions, and the unclarity of questions of propositional identity has sometimes been cited as a reason for denying that there is a proper distinction between propositions and the sentences that express them. Moore 31 once worried whether the proposition that the sun is larger than the moon and the proposition that the moon is smaller than the sun are the same proposition, and he concluded that in one sense they are the same, in another they are not. If those senses could be clearly distinguished, this need not be disturbing, but it does not seem that they can be.

Questions of propositional identity between different verbal formulations are only worth pursuing (when they are) with language users capable of intelligent dialectical discussion as to what they would be saying in using those formulations. Of course "intelligent dialectical discussion" is quite vague, and it can be conceded that satisfying that condition does not guarantee success in the enquiry. The enquiry as to whether the proposition expressed by "Line $L$ is tangent to curve $C$ at point $P$ " and the proposition expressed by "For any point $P^{\prime}$ distinct from $P$ on $C$ and any line $L^{\prime}$ in the same plane as $L$ intersecting $L$ at $P$, there exists an angle measure $m$ such that if the lesser angle of intersection between $L$ and $L^{\prime}$ is less than $m$, then $L^{\prime}$ intersects $C$ at some point between $P$ and $P^{\prime \prime}$, are identical, could be pursued in an intelligent and sincere dialogue without yielding a correct answer. It might be replied that it could not be a very intelligent discussion if it did not get that question answered. But it is being conceded that this may not be so.

However, this could not happen with Moore's examples. An intelligent person might not know whether " $a$ is in relation $R$ to $b$ " and " $b$ is in the converse of the rela- 
tion $R$ to $a$ " expressed the same proposition, but he would have to find this due to failure to understand the terms. This is also true of the four questions whether $M A=M B$, $\mathrm{TA}=\mathrm{TB}, \mathrm{MA}=\mathrm{TA}$ and $\mathrm{MB}=\mathrm{TB}$, provided that the cases sketched here were fully depicted. Intelligent inquirers proceeding in good faith could not fail to see that, in the cases intended here, the first two of those identities are true while the latter two are false. So we return to the problem for the doctrine that there is a property of being present. That doctrine seems to entail that $\mathrm{MA}=\mathrm{MB}$, which is inconsistent with the concessions about identity required above.

One response is to give up the idea that there is such a property as being present and hold that "present" is indexical in the way "this time" is. Another response would be to hold that B more clearly indicates the subject of the assertions and that in all four, $\mathrm{MA}, \mathrm{MB}, \mathrm{TA}$, and $\mathrm{TB}$, the predicate really is, being present, but the subject on Monday is different from the subject on Tuesday.

This latter has the consequence that one could never make a persisting threedimensional object the subject. For A is as well suited for that purpose as any sentence, especially if we recognize that MA and TA could equally well be asserted by use of

C. The sundial is illuminated.

MC and TC must equal MA and TA, respectively, and they are as good candidates of attributing a property to a persisting object as there could be. That is, it is as reasonable as could be to hold that the subject of MC and TC is one and the same persisting sundial. But the effort to hold that the predicate would be the same in each leads the line (presently) under discussion to take B as the guide to the real subject. And that requires taking $\mathrm{MC}$ and $\mathrm{TC}$ to have as real subjects two distinct cases of the dial's being illuminated, rather than one persisting dial.

To the extent that we take seriously this notion of "real subject," it seems that we could not have both the property of presence as a predicate and the persisting threedimensional object as a subject. This would not be to deny that there is a property of being present or that there are persisting three-dimensional objects. But in our assertions we would not have them featured together as predicate and subject, respectively. And this would tempt some to conclude that there really are not things of both kinds. The so-called B theory involves rejecting both kinds.

My view is that there are persisting three-dimensional objects and there is a property of being present which the illumination of the sundial on Monday at noon has at noon "and then" loses. The traditional puzzle as to when the event of the dial illumination loses the property of being present should be answered in the same manner as Zeno's Arrow paradox; that is, there is no instant when the arrow ceases to be located at a point $P$ at instant $t$. There is just a set of instants after the arrow being at $P$ which has no first point, and since the arrow is in motion at $P$, there is also a set before $t$ with no last point. The explanation for the other cases, of a motion ending at a point or beginning at a point are also familiar.

For a durational event, the explanation is the same, except that there is a certain arbitrariness in deciding whether the duration interval is open or closed or open on one end and closed on the other. The dubiousness of giving measurable physical meaning to such distinctions might cause some to suspect the entire explanation. But it is a 
coherent explanation.

The proposition can be represented verbally so as to feature either the persisting object or the property of being present. But both cannot be made out to be the "real" subject and predicate, respectively. My view is that a proposition does not have a "real" subject or predicate. The subject-predicate distinction for sentences does not make sense for propositions. But the looser notion of "about" does apply. Then MA and $\mathrm{MB}$ and $\mathrm{MC}$ are the same proposition, and thus about the same things, but the sentences $\mathrm{A}, \mathrm{B}$, and $\mathrm{C}$ highlight different ones from this cast.

Most important, though, is the fact that our assertion cannot capture the transient property of presence as the predicate of a persisting object. To do that would be to assert a proposition which changes in truth value. We can, of course, make sense of a notion of proposition such that the proposition in that sense is something that changes in truth value, just as some sentences change in truth value. But that is not the kind of proposition which is the topic of the classical law that no proposition is both true and false.

This is not to suggest that there is anything wrong with "Tense Logic." The question is simply whether there is a conception of proposition such that no proposition can change in truth value. So conceived, propositions cannot have as real predicates the property of being present. But it should help make this palatable to recognize that propositions cannot have real predicates at all. The same proposition is representable by a variety of different sentences with different predicates.

We can nonetheless recognize that there is such a property as being present and that time is not a sort of dimension of being, with 'past', 'present', and 'future' merely being indexical references to locations in a block universe. This property is like any other thing that exists, in that it can be predicated of any thing whatever. But if it is not being predicated of an eternal thing, then it is only truly predicated of such a thing as this or that case of the dial's being illuminated.

However, it would seem that the proposition $\mathrm{MB}(=\mathrm{MA}=\mathrm{MC})$ would be true at the time Monday noon's dial illumination is present and would cease to be true at later times. Perhaps there would be vagueness as to the extent of the "case" of illumination, but it would clearly end some time and before Tuesday. So it would seem that the proposition must change in truth value.

This is indeed a powerful appearance. But we should be steadied by considering

D. This case of the sundial's being illuminated is present at this time.

MD is the same proposition as MA, MB, and MC, and it is not a proposition that ever changes in truth value. Asserting $\mathrm{D}$, in the cases under consideration, conveys exactly the same information as A, B, or C. Some assertions of this proposition will be made when the event has the property of being present. But this feature of the assertion cannot be brought into the content as the fixed predicate of such a content. There is no such thing as the fixed predicate of a proposition. Even adding such words as "this assertion is being made in the present" will not accomplish the assertion of a proposition that changes in truth value. What is conveyed is that the assertion is made at that time, and whether that is so does not change. Sundials can change, but not their being in certain conditions at certain times. Those conditions can become present and cease to be present. But the propositions which report those happenings are eternal 
and do not change truth value. They will never feature the property of being present in such a way that the acquisition or loss of that property changes their truth value.

Those who oppose this should then concede that MA and TA are the same proposition after all, since they would on that account, both attribute the same property to the same thing. This is their basis for saying that a proposition can change truth value. MC would be true as long as the sundial is illuminated and would after that be false. They would reject the idea that there is no property of being illuminated simpliciter as opposed to the property of being illuminated at this time, or at that time, or at some time, or at all times. They would concede that this alleged simple property can be chronologized but hold that it can be predicated in itself or also with the (possibly redundant) addition of the property of being present. That is how they would defend the view that propositions such as MA or MC change in truth value while persisting as the same proposition. But then they should concede further that MA does not equal $\mathrm{MB}$ and TA does not equal TB. This is to claim a difference in information where there is none and is logically worse than the B theory.

Propositions such as MB and TB make it seem that we must choose between holding that the ascription of presentness is either an illusion of grammar or else results in propositions which change truth values. This is irresistible if we assume that presentness must be the predicate of the proposition if it is to be at all. Noting that $\mathrm{MD}=\mathrm{MB}$ should help us to renounce this assumption. Presentness cannot be predicated as holding as of the time of predication (and not at all times) without thereby equally predicating a relative presentness, thus yielding a proposition which does not change truth value. This is not unique to presentness but holds equally for all properties which can be gained and lost over time. ${ }^{1}$ Acknowledging this allows us to consistently recognize that there is such a property as being present (thus accepting a crucial A theory doctrine) while denying that propositions ever change in truth value (thus accepting a crucial B theory doctrine).

Different people, speaking at different times, may all use (for just one example) the sentence B with reference to the very same case of the dial's being illuminated to say of that illumination that it occurs at the very same time. Each of their assertions will include information about a different source and time of assertion. What will be a common assertion for all of them will be the proposition, with respect to a certain case of illumination, that it is present as of the time of that case, that this case of the dial's being illuminated is present as of the time of this illumination. One of the speakers may be speaking at the time and place of the illumination and thus recognize that the event is absolutely present. Nonetheless, the content of his assertion, the proposition he asserts, unavoidably attributes presence as of the time of the event, which in his case, is the same time as the time of his assertion. He may recognize the presentness of the assertion perfectly well. But this transitory absolute presentness cannot be the predicate of the proposition which represents the content of his assertion. Nor could it be the common feature of the various assertions, in the way that the proposition that two plus two equals four can be.

This in itself does not explain why we should accept the property of being present. The reason is just that it is this property (and its kindred "A determinations") which keep all times from being equally real. But why try to avoid that consequence? The B theorist has honorable arguments for its being the truth. To claim that we have 
an idea of what it is to be present which we recognize in our experience invites the reply that we get this idea every time we pay attention to our experience. If our idea of being present were merely something common to these experiences then it would be the sort of relative presence congenial to the B theory. If it is not, then we cannot claim to have derived the idea by abstraction from our experience.

This is not a difficulty for those of us who do not view our grasp of properties as obtained by abstraction. Specifically, we recognize the presentness of an event by observing and contrasting its situation with past and future events. We can look out the window and directly observe a place distinct from ours, which is just as real as ours. We cannot directly observe a past or future time. This is not a tautology reflecting merely the idea that "direct observation" means observation of something simultaneous with the observing. The differences among memory, anticipation, and direct observation are introspectively (directly) observable differences in the quality of experience. This is the basis for a difference in meaning among the terms "memory," "anticipation," and "direct observation," rather than the converse.

This argument for the existence of a property of being present can, of course, be rejected, as is exhibited by the B theory. The purpose here is just to make two points clear. First, the reason for thinking that while all places are equally real, this is not true of times, is not that propositions change in truth value. That propositions change in truth value is a doctrine generally associated with the A theory. But (and this is the second point) this is a conflict with classical logic which is not required by the recognition of presentness. Presentness is, as they say, what keeps everything from happening at once. The B theory alternative, a higher dimensional space, strikes many of us as harmless and enlightening as a mathematical model but incredible if taken to entail the real existence of all events in world history at different locations in a higher dimensional manifold.

The first point is not a simple matter of logic and has not been defended adequately here. But the second point is a simple one which has been neglected and is worth emphasizing. It does not follow from the existence of a property of absolute presence that predicating this property yields an assertion whose content changes in truth value. I am presently recognizing that the process of my typing this very sentence is present. That process is now past. But the proposition that I asserted in that typing is timelessly true, like all true propositions. I might just as well have typed out a sentence using the alleged "tenseless" devices favored by the B theorists.

The notion of "tenselessness" has been the object of numerous attempts at explanation. Some may have succeeded, and more than one clear notion may have been associated with this phrase, in spite of the fact that it makes no immediate sense in application to English. One feature of "tenseless translations" of tensed sentences is that they were aimed at bringing out that the content of the assertion was a proposition that does not change in truth value. Thus when I said earlier that my process of typing a certain sentence was present, I meant, by way of assertion, that the process was going on as of a certain time, a certain relative present. Just what relative present it is would require dialogue. One candidate would be the time of that typing. This would make my claim just the claim that there is some time when that sentence is typed. That is some content, but there would be more if more information about that time were brought in. 
However the niceties as to the content of my assertion are filled out, it is in line with classical logic to ensure that the result is not something which changes in truth value. Thus the property of being present does not play the role in my assertion which one might naturally expect it to play. I might as well not have mentioned it, and spoken instead in terms of some relative present. But the impossibility of giving presentness a truth value changing role in an assertion does not entail that there is no such property. It is just not a property which we come to know best by the analysis of assertions. Other kinds of reflection are what convince those of us who are convinced—of its existence.

Acknowledgments I am indebted to Charles Klein and to Gene Mills for helpful discussions on this topic.

\section{NOTE}

1. This is not to assume that all uses of A must predicate presentness as of the time of predication. One can use the narrative present to say that the sundial is illuminated at present and mean only a present in the narrative, without locating that "present" relative to the time of the predication at all. You may be a seer reading the narrative from a crystal ball which only guarantees that the things reported happen at some time or another. Such uses were not the ones intended above in discussing MA-D.

\section{REFERENCES}

[1] Kant, I., Critique of Pure Reason, St. Martin's Press, New York, 1965.

[2] McTaggart, J. M. E., “The unreality of time,” Mind, n.s., vol. 17 (1908), pp. 457-74.

[3] Moore, G. E., "Russell's 'Theory of desciptions'," pp. 175-225 in The Philosophy of Bertrand Russell, edited by P. A. Schilpp, Harper and Row, New York, 1951.

Corcoran Department of Philosophy

University of Virginia

512 Cabell Hall

Charlottesville VA 22903-2443

email: JC7Y@virginia.edu 